\title{
$\alpha$-galactosidase: aspectos gerais e sua aplicação em \\ produtos a base de soja ${ }^{1}$
}

\section{$\alpha$-galactosidase: general aspects and its application on soybean oligosaccharides}

\author{
Silvana Pedroso de Góes² ; Mara Lucia Luiz Ribeiro ${ }^{3 *}$
}

\section{Resumo}

Esta revisão de literatura tem por objetivo apresentar tópicos relativos à $\alpha$-galactosidase, considerando suas características gerais, fontes de obtenção, funções e metodologias utilizadas em sua determinação. Esta enzima, ausente no trato digestivo humano, hidrolisa ligações $\propto-(1,6)$ de oligossacarídeos da família da rafinose. Estes açúcares, chamados flatulentos, são encontrados em muitas leguminosas como a soja, uma rica fonte de proteína. Entretanto, devido à presença desses açúcares, fatores antinutricionais como a flatulência são atribuídos à soja, diminuindo seu consumo. A $\alpha$-galactosidase produzida por alguns microorganismos e vegetais pode ser empregada em alimentos a base de soja para reduzir a interação da flora intestinal com os oligossacarídeos, diminuindo assim a flatulência.

Palavras chave: $\alpha$-galactosidase, oligossacarídeos, soja.

\begin{abstract}
This literature review has the aim to present topics related to $\alpha$-galactosidase, considering its general characteristics, sources, functions and methods used in its determination. This enzyme, lacking in the human digestive tract, hydrolises $\propto-(1,6)$ bonds from oligosaccharides of the raffinose family. These carbohydrates, called flatulents, are found in leguminoses like soybean, a rich source of protein. However, due to the presence of these flatulents sugars, antinutritional factors are attributed to soyben, decreasing its consumption. $\alpha$-galactosidase produced by some microorganisms and vegetables can be used in soybean based foods to reduce the interaction of the intestinal flora with the oligosaccharides, decreasing in this way the flatulence.
\end{abstract}

Key words: $\alpha$-galactosidase, oligosaccharides, soybean

\section{Introdução}

A enzima $\alpha$-D-galactosídeo galactohidrolase (E.C. 3.2.1.22), conhecida como melibiase ou $\alpha$ galactosidase, catalisa a hidrólise de ligações $\alpha-(1,6)-$ galactosídicas, liberando a $\propto$-D-galactose. Estas ligações são encontradas em oligossacarídeos tais como a melibiose, a rafinose e a estaquiose, entre outros. A rafinose, um trissacarídeo e a estaquiose, um tetrassacarídeo (ESKIN et al., 1989; MAITY; PAUL, 1991; RUIZ et al., 1995; MULIMANI; RAMALINGAN, 1997) estão presentes em quantidades significativas na soja (100 a $150 \mathrm{~g} / \mathrm{Kg}$ de peso)

\footnotetext{
1 Parte integrante da monografia apresentada ao Departamento de Bioquímica da Universidade Estadual de Londrina, em 2000, para obtenção do título de Especialista em Bioquímica Aplicada.

2 Aluna do Curso de Especialização em Bioquímica Aplicada.

3 Docente do Departamento de Bioquímica - CCE - Universidade Estadual de Londrina , CP. 6001, CEP: 86.051-990, Londrina - PR

* Autor para correspondência.
} 
(DE REU et al., 1997) e outras leguminosas, tais como feijão e ervilha. São carboidratos de baixo peso molecular, conhecidos como flatulentos, não metabolizáveis no intestino humano devido à ausência de $\alpha$-galactosidase. Entretanto, estes são fermentados pela flora intestinal, principalmente por Clostridium perfringens, provocando flatulência (HYMOWITZ et al., 1972; RACKIS et al., 1974; SILVA; BRAGA, 1982; SOMIARI; BALOGH, 1992; RUIZ et al., 1995; MANZANARES; GRAAFF; VISSER, 1998). Esta pode ser acompanhada de diarréias, dores de cabeça, dispepsia, confusão mental e diminuição da capacidade de concentração no trabalho (RUIZ; ACOSTA; CISNEIROS, 1995; SANNI; ONILUDE; OGUNDOYE, 1997). Estes distúrbios gastrointestinais contribuem para o baixo consumo da soja, um alimento rico em proteínas $(380 \mathrm{~g} / \mathrm{K}$ de peso seco) (MOHAMED; MEBRAHTU; RANGAPPA, 1991; PORTER et al., 1992; DE REU et al., 1997) e que pode ser usado como alternativa para proteína animal.

No intuito de aumentar a aceitabilidade da soja na alimentação, vários métodos são usados para a redução do teor destes oligossacarídeos: o tratamento enzimático (CRUZ et al., 1981; SAKAI et al., 1987; SHIVANNA; RAMAKRISHINA; RAMADOSS, 1989; MAITY; PAUL, 1991; RUIZ; ACOSTA; CISNEIROS, 1995; MULIMANI; RAMALINGAN, 1997), a hidratação dos grãos (MULYOWIDARSO; FLEET; BUCKE, 1991; MULIMANI; RAMALINGAN, 1997; SANNI et al., 1997), o cozimento (SILVA; BRAGA, 1982; MULIMANI; THIPPESWAMY; RAMALINGAN, 1997), a fermentação (SUPARMO, 1987; REHMS; BARZ, 1995), a germinação (REDDY; SALUNKHE, 1980; ABDULLAH; BALDWIN; MINER, 1984; YAKLICH, 1985; BAU et al., 2000). Entre estes, tratamento enzimático tem sido o mais indicado, por sua especificidade e condições brandas de processamento (RUIZ; ACOSTA; CISNEROS, 1995).
Assim, esta revisão de literatura fornece algumas informações sobre a $\alpha$-galactosidase, considerando suas características gerais, fontes de obtenção, funções e metodologias utilizadas em sua determinação.

\section{A $\alpha$-galactosidase}

Nas plantas, a $\alpha$-galactosidase apresenta diferentes funções, estando presente em todos os tecidos que contém $\alpha$-galactosídeos (ALANI; SMITH, MARKAKIS, 1989). Em hipótese, esta pode atuar como pesticida natural, pois se trata de uma lectina, e algumas lectinas são proteínas tóxicas de reserva (PORTER; LADISCH , 1992).

Segundo Dopico, Nicolás e Labrador (1989), na parede celular das plantas ocorrem reações enzimáticas que promovem a hidrólise de glucanos e a quebra das ligações de seus polissacarídeos. Esta é uma característica da autólise para promoção do crescimento das plantas. Este fato foi confirmado no estudo de Pisum sativum, com a participação da $\alpha$-galactosidase neste processo para o crescimento da planta.

A $\alpha$-galactosidase atua no reconhecimento celular, no transporte de açúcares e como glicoproteína na organização de sistemas multi-enzimáticos. Pode, também, agir como antibiótico contra as bactérias do solo, protegendo a planta contra substâncias $\alpha$ galactosídicas fitotóxicas produzidas por microrganismos invasores como Didymella applanta que infecta framboeseiras (apud PORTER et al., 1992).

Esta enzima apresenta ainda diversas aplicações tais como, estudo da estrutura molecular de açúcares; melhoramento da sacarose extraída de beterraba para diminuir o teor de rafinose, já que esta impede a cristalização da sacarose (ANNUNZIATO; MAHONEY; MUDGETT 1986; MULIMANI; RAMALINGAM, 1995); eliminar o sabor desagradável de feijão cru do leite de soja, além de desodorizá-lo (RUIZ; ACOSTA; CISNEIROS, 1995) e em produtos à base de soja diminuir o teor dos oligossacarídeos que causam flatulência (CRUZ; PARK, 1982). 


\section{Fontes da enzima e suas características}

A $\alpha$-galactosidase pode ser obtida a partir de bactérias, fungos ou vegetais. Procurando otimizar a produção e obtenção desta enzima, estas diferentes fontes têm sido estudadas (SHIVANNA, RAMAKRISHNA; RAMADOSS, 1989). Segundo Manzanares, Graaff e Visser (1998), a enzima de origem bacteriana apresenta vantagens sobre a enzima de origem fúngica, uma vez que sua produção é maior. No entanto, galactosidases de fungos são obtidas mais facilmente devido à sua localização extracelular e seu amplo perfil de estabilidade, sendo portanto viável sua produção em grande escala (ANNUNZIATO; MAHONEY; MUDGETT, 1986; SOMIARI; BALOGH, 1992).

Segundo Alani, Smith e Markakis (1989), esta enzima apresenta diferentes formas, podendo ser monomérica ou tetramérica, com termo-estabilidade e conformação diretamente relacionadas (PORTER et al., 1992).

A maioria das --galactosidases produzida por fungos é glicoproteínas, sendo encontrados resíduos de glucose e manose na enzima da soja (PORTER et al., 1992; ZEILINGER et al., 1993). Na soja, a galactose protege parcialmente o sítio catalítico da enzima, em sua forma tetramérica, contra a desnaturação pelo calor, sendo esta proteção pH-dependente.

Quanto à enzima de origem vegetal, Mulimani e Ramalingam (1997) realizaram experimentos com farinha de soja e chegaram à conclusão de que a $\alpha$ galactosidase de guar (Cyamopsis tetragonolobus) atuou melhor na redução dos oligossacarídeos que a enzima de origem microbiana.

\section{Enzima de origem bacteriana}

Sakai et al. (1987) em estudos com Bifidobacterium breve em leite de soja observaram redução de rafinose e estaquiose, sendo a atividade máxima da enzima a $50{ }^{\circ} \mathrm{C}, \mathrm{pH}$ 5,0. Estas condições, dadas como ótimas para a atuação da enzima, assemelh $\alpha$-se às descritas para a enzima produzida por Bifidobacterium longum e para Lactobacillus fermentum (GARRO et al., 1994; GARRO et al., 1996). Entretanto, diferenças no comportamento destas enzimas são percebidas quanto à ação de inibidores: a adição de par $\alpha$ cloromercuribenzoato (PCMB) afetou a atividade enzimática de $B$. breve e $L$. fermentum sugerindo a presença de grupos sulfidrilas no sítio catalítico da enzima, enquanto que $\alpha$-galactosidase produzida por B. longum não sofreu alterações com a adição de PCMB, revelando diferenças estruturais nas enzimas produzidas por organismos diferentes. A adição de íons $\mathrm{Hg}^{2+}$ inibiu a atividade das enzimas produzidas pelos três microrganismos, provavelmente devido à presença de grupos tiol.

Porter et al. (1992) destacou o uso de $\alpha$-galactosidase produzida por Bacillus stearothermophilus como estável a temperaturas entre $65^{\circ}$ e $70{ }^{\circ} \mathrm{C}$ sem a necessidade de indutores. Isto representa uma excelente fonte da enzima, pois quanto maior a temperatura de atuação da enzima, melhor será seu emprego em alimentos sendo mais resistente a tratamentos térmicos. Estudos semelhantes foram realizados por Eveleigh (2000) com a bactéria Thermotoga neopolitana, levando a conclusão de que seu emprego na alimentação é viável.

\section{Enzima de origem fúngica}

Cruz, Batistela e Wosiacki (1981) utilizaram Saccharomyces cerevisiae para fermentar a soja modificando seu teor de açúcares para que Cladosporium cladosporioides pudesse produzir $\alpha$-galactosidase e hidrolisar os oligossacarídeos ali existentes. Os substratos liberados foram utilizados pela enzima, que se demonstrou estável em temperaturas de $55^{\circ}$ a $60{ }^{\circ} \mathrm{C}$, proporcionando redução de $50 \%$ nos níveis de açúcares da família da rafinose.

Observando a necessidade de uma fonte de carbono no meio de cultivo para o desenvolvimento de microrganismos, Ruiz, Acosta e Cisneiros (1998) testaram o uso de resíduos liberados durante a produção de leite de soja para o crescimento de Aspergillus orizae, obtendo aumento na produção da enzima pelo 
fungo. A $\alpha$-galactosidase de $A$. orizae apresenta máxima atividade a $50{ }^{\circ} \mathrm{C}, \mathrm{pH}$ 4,0 (CRUZ; PARK, 1982). Além de produzir $\alpha$-galactosidase, este fungo produz invertase ( $\beta$-D-fructofuranosídeo fructohidrolase, E.C. 3.2.126), cuja combinação hidrolisa mais eficientemente os galactooligossacarídeos, convertendo-os a monossacarídeos (DELENTE; LANDERBUR, 1972). Entretanto, a presença de invertase afeta a qualidade da sacarose e, portanto, sua presença em preparações de $\alpha$ galactosidase deve ser evitada (RUIZ; ACOSTA; CISNEIROS 1995). Absidia sp, Penecillium duponi, Mortierella vinacea e Saccharomyces sp são produtores de $\alpha$-galactosidase livres de invertase (DEKKER, 1994).

Somiari e Balogh (1992), em estudos com Aspergillus niger, observaram que a $\alpha$-galactosidase produzida pelo fungo reduziu os teores de estaquiose e rafinose de feijão caupi em $82 \%$ e $95 \%$, respectivamente, com atividade máxima a $50{ }^{\circ} \mathrm{C}, \mathrm{pH} 5,0$ sendo estável por 3 meses a $-20{ }^{\circ} \mathrm{C}$ (SOMIARI; BALOGH, 1993). Segundo Manzanares, Graaff e Visser (1998), A. niger produz dois tipos de $\alpha$ galactosidases capazes de hidrolisar oligossacarídeos flatulentos. As duas frações da enzima apresentam peso molecular estimado por SDS-PAGE de $72 \mathrm{e}$ 69Kda, de caráter ácido, com pI = 4,2 e 4,6.

Ao testar o crescimento de A. niger sobre trigo e arroz, Somiari e Balogh (1993) observaram que o melhor crescimento e consequentemente melhor atividade enzimática se deu sobre o trigo. A enzima se manteve estável por 1 hora em temperatura de $35^{\circ}$ a $50{ }^{\circ} \mathrm{C}$, sugerindo que compostos do trigo conferem proteção à enzima contra a desnaturação pelo calor, sendo esta proteção $\mathrm{pH}$-dependente. Quanto à proteção, fato semelhante ocorre em Vicia faba e em soja, sendo a galactose protetora da $\alpha$-galactosidase em sua forma monomérica e tetramérica, respectivamente. Segundo Ruiz Acosta e Cisneiros (1995) A. niger constitui uma excelente fonte de $\alpha$ galactosidase para emprego em alimentos, pois é considerado um microrganismo GRAS (generally recognized as safe). Entretanto, sua intensa esporulação observada após o terceiro e quarto dias de crescimento em meio de fermentação sólida, propicia o desenvolvimento de cor, sendo indesejável para o ensaio enzimático e para o processamento de alimentos.

Ao estudar resíduos de soja e de can $\alpha$-de-açúcar como indutores para a produção de $\alpha$-galactosidase por Monascus grown, Wong-Leung, Fong e Lam (1993) obtiveram melhor produção da enzima em resíduos de cana, uma vez que estes apresentam cerca de 10 vezes mais carboidratos que os resíduos de soja. Entretanto, melhor crescimento do micélio foi observado ao utilizar resíduos de soja devido ao elevado teor de nitrogênio presente neste substrato. Ao combinar em meio aquoso $5 \%$ de resíduos de soja e $7 \%$ de resíduo de cana, houve um ótimo crescimento do fungo e ótima produção da enzima. $M$. grown é um fungo usado no extremo Oriente como componente de vinho tinto e em alimentos fermentados de soja, sendo muito estudados por possuir pigmentos vermelhos passíveis de uso em alimentos. Observando os resultados, os pesquisadores sugerem a obtenção de $\alpha$-galactosidase como um co-produto durante o isolamento dos pigmentos.

Conforme Rehms e Barz (1995), Rhizopus fungi também é produtor de $\alpha$-galactosidase. Entretanto, para a hidrólise completa de galacto-oligossacarídeos da soja, é necessária a ação conjunta de duas enzimas: a $\alpha$-galactosidase que degrada as ligações entre galactose e sacarose e a b-frutosidase que hidrolisa as ligações entre frutose e glicose presentes na sacarose, rafinose e estaquiose.

Zeilinger et al. (1993) estudando a $\alpha$ galactosidase produzida pelo fungo Trichoderma reesi, verificou que este fungo é capaz de crescer e produzir a enzima utilizando lactose como fonte de carbono. Possivelmente esta enzima pode ser induzida por polímeros ou oligômeros contendo ligações $\alpha$-galactosídicas terminais e também por monômeros de galactose, sendo interessante, pois é possível que a galactose livre não esteja amplamente disponível no habitat natural do fungo. A ativida- 
de enzimática aumentou com o aumento da biomassa, mas curiosamente a eficiência na produção diminuiu. As condições ótimas encontradas para a enzima foram de $\mathrm{pH} 4,0$ e $60{ }^{\circ} \mathrm{C}$. A enzima foi estável por 12 horas em $\mathrm{pH}$ que variou de 4,5 a 6,5.

Ao testar a capacidade hidrolítica de $\alpha$ galactosidase produzida por Gibberella fujikuroi na farinha de grãos-de-bico (Cicer arietinum), redução de $91 \%$ de rafinose e $82 \%$ de estaquiose + verbascose foi observada quando a enzima foi incubada por duas horas e meia. A $\alpha$-galactosidase é um metabólito secundário produzido pelo fungo quando este cresce em condições submersas, tendo como fonte de carbono a rafinose ou melibiose, produzindo como metabólito primário o ácido giberélico. A enzima é secretada e fica presa ao micélio, sendo a filtração o método indicado para separá-la do ácido giberélico. Consequentemente o custo de produção em larga escala é baixo (MULIMANI; RAMALINGAN, 1997).

Vincent et al. (1999) em trabalhos com Saccharomyces cerevisiae concluíram que o melhor crescimento da levedura e produção de $\alpha$ galactosidase se deu em melaço de can $\alpha$-de-açúcar ou de beterraba. Ruiz, Acosta e Cisneiros (1995), porém sugerem que clones de Saccharomyces $s p$ expostos à luz ultravioleta aumentaram sua produção de $\alpha$ galactosidase sem necessidade de fonte de carbono.

\section{Enzima de origem vegetal}

Quanto à $\alpha$-galactosidase vegetal, Mujer, Ramirez e Mendonza (1984), em estudos com coco, observaram que a atividade da enzima $\alpha$-galactosidase aumenta com a idade da planta e sua principal função é degradar as galactomananas de reserva das sementes no início da germinação.

Shivanna, Ramakrishina e Ramadoss (1989), realizou experimentos com guar e observou que a $\alpha$ galactosidase produzida pela planta hidrolisou completamente a rafinose e cerca de $80 \%$ da estaquiose, em 30 minutos. Além disso, as condições ótimas para a atuação da enzima (pH 5,0 e 50 ${ }^{\circ} \mathrm{C}$ ) abriram boas perspectivas para seu emprego no leite de soja, além de constituir uma fonte barata de obtenção da enzima. Resultados semelhantes levaram Mulimani e Ramalingan (1997) à conclusão de que a $\alpha$ galactosidase de guar atua melhor hidrolisando oligossacarídeos da farinha de soja que a de fonte microbiana.

Bhaskar, Ramachandra e Virupasha (1990) realizaram experimentos com Cassia sericea e observaram a produção de duas formas de $\alpha$-galactosidase pela planta. Ambas apresentaram atividade ótima em $\mathrm{pH} 5,0$ e $50^{\circ} \mathrm{C}$. Ao serem testadas quanto à presença de inibidores, houve inibição total das enzimas quando estas foram colocadas junto a íons $\mathrm{Cu}^{2+}, \mathrm{Hg}^{2+}$ e $\mathrm{Ag}^{+}$, bem como na presença de PCMB. Já a iodoacetamida e o 2-mercaptoetanol provocaram inibição parcial da enzima.

Chrost e Schmitz (1997) realizaram pesquisas com $\alpha$-galactosidases encontradas em melão (Cucumis melo). Segundo estes pesquisadores, plantas da família das Cucurbitaceas apresentam 2 tipos de $\alpha$-galactosidases, uma com atividade em $\mathrm{pH}$ ácido e outra com atividade em $\mathrm{pH}$ alcalino, sendo a distribuição e função dessas enzimas, dependentes do desenvolvimento e dos tecidos da planta. A atividade mais alta da enzima foi detectada na fruta madura. Da clivagem da rafinose pela enzima resultam sacarose e galactose. A galactose é tóxica para a planta e é então rapidamente utilizada para a biossíntese de mais sacarose, pois esta representa energia metabolizável para a planta.

\section{Métodos usados na investigação da $\alpha$-galactosidase}

\section{Extração da enzima}

A extração da enzima, a partir das diferentes fontes produtoras, pode ser realizada através de diferentes métodos. 
Para extrair a $\alpha$-galactosidase de sementes, Barham et al. (1971) incubaram farinha resultante da moagem destas em tampão McIlvaine e após centrifugação utilizaram o sobrenadante no ensaio enzimático.

Cruz, Batista e Wosiacki (1981) filtraram o micélio de Cladosporium cladosporioides em papel de filtro lavado com $\mathrm{KCl}$, centrifugaram e usaram o sedimento como enzima intracelular e o sobrenadante como enzima extracelular .

Mujer, Ramirez e Mendonza (1984) congelaram e homogeneizaram endosperma de coco em tampão acetato de sódio, centrifugando e utilizando o precipitado para o ensaio enzimático.

Shivanna, Ramakrishna e Ramadoss (1989) obtiveram amostras de guar a partir da trituração das sementes congeladas e homogeneizadas em acetona. As amostras foram incubadas em tampão fosfato de sódio com 2-mercaptoetanol e PMSF insolúvel. $\mathrm{O}$ extrato foi filtrado e centrifugado. Após saturação com sulfato de amônio e centrifugação, o precipitado foi misturado a glicerol e dializado. A enzima obtida foi usada no ensaio enzimático.

A enzima de caupi foi extraída por Alani, Smith e Markakis (1989) com tampão Mcllvaine e após a centrifugação e saturação, o precipitado foi coletado por nova centrifugação e ressuspenso em tampão McIlvaine e em seguida dializado. A fração obtida pela diálise foi aplicada em uma coluna de Sephadex G-100 e as duas frações eluídas passaram por ultracentrifugação usando para isso uma membrana Amicon $\mathrm{YM}_{10}$.

Chrost e Schimitz (1997) congelaram amostras de tecido de melão em nitrogênio líquido e a farinha fina obtida foi incubada em tampão acetato de sódio e PVPP (polivinilpolipirrolidona) 2\%. Após centrifugação a $30.000 \times$ g por 30 minutos, o sobrenadante foi coletado para a realização do ensaio enzimático.

Kuo, Lowell e Smith (1997) realizou a extração enzimática de sementes de soja em temperaturas entre $0^{\circ}$ e $5^{\circ} \mathrm{C}$. A farinha obtida pela moagem das sementes foi homogeneizada com HEPES-NaOH, DTT e $\mathrm{MgCl}_{2}$ e centrifugada. O sobrenadante foi novamente incubado no mesmo tampão e passado pela coluna Excellulose GF-5 2,5.

\section{Purificação da enzima}

Geralmente, a purificação de $\alpha$-galactosidase de sementes de soja consiste num procedimento que varia de cinco a seis etapas, sendo elas a crio-precipitação, a precipitação ácida, o fracionamento por sulfato de amônio seguido por duas ou três etapas cromatográficas (PORTER; LADISCH, 1992).

\section{Ensaio enzimático}

A atividade enzimática da --galactosidase tem sido determinada espectrofotometricamente por diversos autores com o uso de p-NPG (para-nitrofenil $\alpha$-Dgalactopiranosídeo) que atua como substrato para a enzima produzindo p-NP (para-nitrofenol), um composto que desenvolve cor e que pode ser medido por espectrofotometria.

O p-NPG foi utilizado para determinar a atividade da $\alpha$-galactosidase de fontes microbianas, como por exemplo a enzima produzida por Lactobacillus fermentum (GARRO et al., 1996), de fontes fúngicas como a de Aspergillus niger (MANZANARES; GRAAFF, VISSER, 1998) ou ainda de origem vegetal como a obtida de sementes de soja (KUO; LOWELL; SMITH, 1997), a de guar (SHIVANNA; RAMAKRISHNA, 1989) e a de frutos de melão (CHROST; SCHIMITZ, 1997).

Para o ensaio enzimático, a enzima é incubada com o substrato (p-NPG) em uma solução tampão, com tempo, temperatura e $\mathrm{pH}$ variáveis em função deste tampão. A reação é finalizada pela adição de carbonato de sódio e o produto formado é quantificado por leituras espectrofotometricas, de 400 a 410 nm (MUJER; RAMIREZ; MENDONZA, 1984; KUO; LOWELL; SMITH, 1997). 
Para a enzima diluída em tampão acetato de sódio, Kuo, Lowell e Smith (1997) conduziram a reação em pH 5,0 a $37^{\circ} \mathrm{C}$, por 30 minutos. Garro et al. (1996) utilizaram tampão McIlvaine $\mathrm{pH} 5,8$ e $45^{\circ} \mathrm{C}$ por 15 minutos.

Devido às variações de tempo, temperatura e $\mathrm{pH}$ encontrados na literatura, sugere-se a realização de uma curva padrão para determinar as condições ótimas da enzima, pois conforme Barham et al. (1971) dependendo da origem, a enzima apresenta particularidades como $\mathrm{pH}$, temperatura e atividade enzimática.

Outro método para avaliação da atividade enzimática utilizado baseia-se na hidrólise da melibiose. Somiari e Balogh (1995) incubaram melibiose $1 \%$ diluída em tampão acetato com o filtrado obtido da massa fermentada de $A$. niger, a $40{ }^{\circ} \mathrm{C}$ por 60 minutos e para finalizar a reação a temperatura foi aumentada para $100{ }^{\circ} \mathrm{C}$ por 10 minutos. A quantidade de glucose liberada foi medida seguindo o método descrito por Sugimoto e Van-Buren.

Cruz, Batistela e Wosiacki (1981) verificaram a atividade enzimática da $\alpha$-galactosidase produzida por C. cladosporioides. Utilizaram como substrato p-NPG e determinaram a glucose formada a partir da hidrólise de melibiose, usando um cromógeno glucose oxidaseperoxidase, conforme Hough e Jones. A enzima foi incubada com solução de melibiose em tampão McIlvaine a $40{ }^{\circ} \mathrm{C}$ por 90 minutos.

\section{Conclusão}

Entre os fatores antinutricionais da soja, os açúcares flatulentos têm sido estudados em todo o mundo na tentativa de minimizar seus efeitos indesejáveis e com isso promover o aumento do consumo de soja e seus derivados, tendo em vista o valor nutricional desses alimentos. Uma alternativa viável para a redução destes açúcares é a utilização da $\alpha$-galactosidase.

Como foi possível observar ao longo desse trabalho, diversas são as fontes produtoras desta enzima. Esta hidrolisa eficientemente as ligações galactosídicas presentes nos oligossacarídeos flatulentos e, a julgar pela grande diversidade de organismos existentes, muitos estudos ainda devem ser realizados na caracterização de outros possíveis organismos produtores da enzima.

Além disso, esforços devem ser realizados também para otimizar a produção da enzima com o emprego de meios de cultivo alternativos para fontes microbianas, como por exemplo o uso de resíduos. Na maioria das vezes, estes representam desperdícios financeiros para as indústrias, além de serem em sua grande maioria poluidores do ambiente.

Com esta revisão bibliográfica, concluímos que o método enzimático apresenta os melhores resultados quanto a redução dos açúcares flatulentos e que o emprego da enzima em alimentos à base de soja é viável. No entanto, no caso de algumas fontes produtoras da enzima mais estudos devem ser realizados para eliminar qualquer dúvida quanto a ausência de toxicidade para o consumidor.

\section{Referências}

ABDULLAH, A.; BALDWIN, R. E.; MINOR, H. Germination effects on flatus-causing factors and antinutrients of mungbeans and two strains of smallseeded soybeans. Journal of Food Protection, Des Moines, v.47, n.6, p.441-444, 1984.

ALANI, S. R.; SMITH, D. M.; MARKAKIS, P. $\alpha-$ galactosidases of Vigna unguiculata. Phytochemistry, New York, v.28, n.8, p.2047-2051, 1989.

ANNUNZIATO, M. E.; MAHONEY, R. R.; MUDGETT, R. E. Production of $\alpha$-galactosidase from Aspergillus oryzae grown in solid state culture. Journal of Food Science, Chicago, v.51, n.5, p.1370-1371, 1986.

BARHAM, D. et al. Studies on the distribution of $\alpha$ galactosidases in seeds. Phytochemistry, New York, v.10, p.1759-1763, 1971.

BAU, H.M et al. Effects of soybean (Glycine max) germination on biologically active components and nutritional value of seeds and biological characteristics in rats. In: INTERNATIONAL SYMPOSIUM ON THE ROLE OF SOY IN PREVENTING AND TREATING CHRONIC DISEASE, $2^{\text {th }}, 1996$, Brussells. Disponível 
em: <www.soyfoods.com/symposium/pa14.html>. Acesso em: 18 jan. 2000.

BHASKAR, B.; RAMACHANDRA, G.; VIRUPAKSHA, T. K. Alpha-galactosidase of germinating seeds of Cassia sericea. Journal of Food Biochemistry, Westport, v.14, p.45-49, 1990.

CHROST, B. ; SCHIMITZ, K. Changes in soluble sugar and activity of $\alpha$-galactosidases and acid invertase during muskmelon (Cucumin melo) fruit development. Journal of Plant Physiology, Stuttgart, v.151, p. 41-50, 1997.

CRUZ, R.; BATISTELA, J. C.; WOSIACKI, G. Microbial $\alpha$-galactosidase for soymilk processing. Journal of Food Science, Chicago, v.46, p.1196-1200, 1981.

CRUZ, R.; PARK, Y. K. Production of fungal $\alpha$ galactosidase and its application to the hydrolysis of galactooligosaccharides in soybean milk. Journal of Food Science, Chicago, v.43, p.1973-1975, 1982.

DEKKER, R. F. H. Enzymes in food and beverage processing.2. Food Australia, North Sydney, v.46, n.4, p.179-181, 1994.

DELENTE, J. ; LANDENBUR, K. Quantitative determination of the oligosaccharides in defaulted soybean meal by gas-liquid chromatography. Journal of Food Science, Chicago, v.37, p.372-274, 1972.

DE REU, J. C. et al. Consistency, polysaccharidase activities and non-starch polysaccharides content of soya beans during tempe fermentation. Journal of the Science of Food and Agriculture, London, v.73, n.3, p.357-363, 1997.

DOPICO, B.; NICOLÁS, G.; LABRADOR, E. Partial purification of cell wall $\alpha$-galactosidases and aarabinosidases from Cicer arietinum epicotyls. Relationship with cell wall autolytic processes. Physiologia Plantarum, Copenhagen, v.75, p.465-468, 1989.

ESKIN, N. A. M. et al. A study of oligosaccharides in a select group of legumes. Canadian Institute of Food Science and Technology Journal, Ottawa, v.13, n.1, p.4042, 1989.

EVELEIGH, D. E. Microbial transformation of plant polysaccharides; hyperthermophilic enzymes; conservation of antique manuscripts. Disponível em: $<$ http://aesop.rutgers.edu/ dougeleleigh.html>. Acesso em: 20 jan. 2000.

GARRO, M. et al. $\alpha$-D-galactosidase (E.C. 3.2.1.22) from Bifidobacterium longum. Letters in Applied Microbiology, Oxford, v.19, n.1, p.16-19, 1994.

GARRO, M. et al. Purification of $\alpha$-galactosidase from Lactobacillus fermentum. Journal of Biotechnology, Amsterdam, v.45, p.103-109, 1996.
HYMOWITZ, T. et al. Variation in the intestinal gasforming sugars in peanut cultivars. Crop Science, Madison, v.12, p.710-711, 1972.

KUO, T. M.; LOWELL, C. A.; SMITH, P. T. Changes in soluble carbohydrates and activities in maturing soybean seed tissues. Plant Science, Amsterdam, v.125, p.1-11, 1997.

MAITY, T. K. ; PAUL, S. C. Low-oligosaccharide soy milk: application of alpha-galactosidase for hydrolysing soy-oligosaccharide. Indian Dairyman, New Delhi, v. 43, n.10, p.443-448, 1991.

MANZANARES, P.; GRAAFF, L.H.; VISSER, J. Characterization of galactosidases from Aspergillus niger : purification of a novel $\alpha$-galactosidase activity. Enzyme and Microbial Technology, New York, v.22, p.383-390, 1998.

MOHAMED, A . I. ; MEBRAHTU, T.; RANGAPPA, M. Nutrient composition and anti-nutritional factors in selected vegetable soybean (Glicine max [L.] Merr.). Plant Foods for Human Nutrition, Dordrecht, v.41, p.89-100, 1991.

MUJER, C. V.; RAMIREZ, D. A.; MENDONZA, E. M. T. $\alpha$-D-galactosidase deficiency in coconut endosperm: its possible pleiotropic effects in makapuno. Phytochemistry, New York, v.23, n.4, p.893-894, 1984.

MULIMANI, V. H. ; RAMALINGAN. Enzymic hydrolysis of raffinose and stachyose in soymilk by alphagalactosidase from Gibberella fujikuroi. Biochemistry and Molecular Biology International, Marrickville, v.36, n. 4, p.897-905, 1995.

. Enzymatic degradation of raffinose family sugars in chickpea flour. World Journal of Microbiology \& Biotechnology, Oxford, v.13, p.583-585, 1997.

MULIMANI, V. H.; THIPPESWAMY, S.; RAMALINGAM. Enzymatic degradation of oligosaccharides in soybean flours. Food Chemistry, Oxford, v.59, n.2, p.279-282, 1997.

MULYOWIDARSO, R. K. ; FLEET, G. H. ; BUCKLE, K. A. Changes in the concentration of carbohydrates during the soaking of soybeans for tempe production. International Journal of Food Science and Technology, Oxford, v.26, p.595-606, 1991.

PORTER, J. E. et al. Effect of $\mathrm{pH}$ on subunit association and heat protection of soybean $\alpha$-galactosidase. Enzyme Microbial Technology, New York, v.14, p.609-613, 1992.

PORTER, J. E.; LADISCH, M. R. Ion-exchange and affinity chromatography costs in $\alpha$-galactosidase purification. Biotechnology and Bioengineering, New York, v.39, p.717-724, 1992.

RACKIS, J. J. et al. Problems encountered in measuring trypsin inhibitor activity of soy flaw. Cereal Science Today, Minneapolis, v.19, p.513-516, 1974. 
REDDY, N. R.; SALUNKHE, D. K. Changes in oligosaccharides during germination and cooking of black gram and fermentation of black gram/rice blend. Cereal Chemistry, Saint Paul, v.57, p.354-360, 1980

REHMS, H.; BARZ, W. Degradation of stachyose, raffinose, melibiose and sucrose by different tempeproducing Rhizopus fungi. Applied Microbiology \& Biotechnology, Oxford, v. 44, p.47-52, 1995.

RUIZ, A.; ACOSTA, M.; CISNEROS, F. Utilizacion de enzimas en los procesos de obtencion de leche y derivados de la soya. Alimentaria, Madrid, v.33, p.127-132, 1995.

Efecto estimulador del licor de remojo del frijol de soya en la produccion de la enzima $\alpha$-galactosidasa. Alimentaria, Madrid, v.36, p.103-106, 1998.

SAKAI, K. et al. Hidrolysis of $\alpha$-D-Galactosyl Oligosaccharides in Soymilk by $\alpha$-D-Galactosidase of Bifibobacterium breve 203. Agricultural Biological Chemistr, Building, v.51, n.2, p.315-322, 1987.

SANNI, A . I.; ONILUDE, A. A.; OGUNDOYE, O . R. Effect of bacterial galactosidase treatment on the nutritional status of soybean seeds and its milk derivative. Nahrung: Chemie, Biochemie, Mikrobiologie, Technologie, Ernaehrung, Berlim, v.41, p.18-21, 1997.

SHIVANNA, B. D.; RAMAKRISHNA, M.; RAMADOSS, C. S. Enzymic hydrolysis of raffinose and stachyose in soybean milk by $\alpha$-galactosidase from germinating guar (Cyamopsis tetragonolobus). Process Biochemistry, London, v.24, p.197-201, 1989.

SILVA, H. C.; BRAGA, G. L. Effect of soaking and cooking on the oligosaccharide content of dry beans (Phaseolus vulgaris, L.). Journal of Food Science, Chicago, v.47, p.924-925, 1982.

SNYDER, H. E. ; KNOW, P. W. Soybeans utilization. New York: AVI Buck, 1987.
SOMIARI, R. I. ; BALOGH, E. Hydrolysis of raffinose and stachyose in cowpea (Vigna unguiculata) flour, using $\alpha$-galactosidase from Aspergillus niger. World Journal of Microbiology and Biotechnology, Oxford, v.8, p.564566, 1992.

Effect of soaking, cooking and crude $\alpha$ galactosidase treatment on the oligosaccharide content of cowpea flours. Journal of the Science of Food and Agriculture, London, v.61, p.339-343, 1993.

Properties of a extracellular glycosidase of Aspergillus niger suitable for removal of oligosaccharides from cowpea meal. Enzyme and Microbial Technology, New York, v.17, p.311-316, 1995.

SUPARMO, Markakis P. Tempeh prepared from germinated soybeans. Journal of Food Science, Chicago, v.52, n.6, p.1736-1737, 1987.

VINCENT, S. F. et al. Comparison of melibiose utilizing backer's yeast strains produced by genetic engineering and classical breeding. Letters in Applied Microbiology, Oxford, v.28, p.148-152, 1999.

WONG-LEUNG, Y. L.; FONG, W. F.; LAM, W. L. Production of $\alpha$-galactosidase by Monascus grown on soybean and sugarcane wastes. World Journal of Microbiology and Biotechnology, Oxford, v.9, p.529533, 1993.

YAKLICH. R. W. Effect of aging on soluble oligosaccharide content in soybean seeds. Crop Science, Madison, v.25, p.701-704, 1985.

ZEILINGER, S. et al. Conditions of formation, purification and characterization of an $\alpha$-galactosidase of Trichoderma reesi RUT C-30. Applied and Environmental Microbiology, Washington, v.59, n.5, p.1347-1353, 1993. 
